Advances in Dynamical Systems and Applications.

ISSN 0973-5321, Volume 16, Number 1, (2021) pp. 159-170

(C) Research India Publications

https://dx.doi.org/10.37622/ADSA/16.1.2021.159-170

\title{
Neuromodeling in Irrigation Management for Sustainable Agriculture
}

\author{
Dmitrii Soloviov ${ }^{1}$, Galina Kamyshova ${ }^{1}$, Viktor Korsak ${ }^{1}$, \\ Nadezhda Terekhova ${ }^{1}$, and Dmitrii Kolganov ${ }^{1}$ \\ ${ }^{1}$ N.I. Vavilov Saratov State Agrarian University, 410012 Teatralnaya Square 1, Russia
}

\begin{abstract}
The article presents the results of research on possibility and efficiency of introducing neuromodeling in irrigation control systems. One of the most effective methods of reducing water supply, saving irrigation water and, as a consequence, the sustainability of agriculture, is the use of differentiated irrigation cycles. However, traditional approaches based only on the physical modeling of processes and relationships, on the one hand, often make it difficult to find effective solutions, and on the other, are difficult to put irrigation into practice. New data mining tools provide improved accuracy and simplicity of implementation by resolving complex relationships in large amounts of parameters and have great potential. In this regard, it seems appropriate to use methods of neural network data analysis. An approach based on a data mining model is proposed, namely a Kohonen neural network clustering model and GIS technologies.
\end{abstract}

\section{INTRODUCTION}

Effective use of agricultural land is the basis for sustainable agricultural development [1]. Among the basic principles of nature management, ensuring the harmonization of the development of natural systems and human societies, the principle of necessary diversity and the principle of balance are of special importance in irrigation reclamation. The principle of the necessary diversity is that an efficient and environmentally safe man-made control system must be as diverse as a controlled natural system. The second principle is the requirement of conformity of economic activity in the developed territory to resource and ecological possibilities of natural systems. Design of irrigation regimes must achieve compliance with both principles, which is ensured by their (cycles) differentiation by the depth of the calculated soil layer and the pre-flood moisture threshold. Such differentiated irrigation cycles that consider the different needs of irrigated crops in water in different phases of growth and development have already been developed and are widely used in the Saratov Volga

\footnotetext{
${ }^{1}$ Corresponding author: gkamichova@mail.ru
} 
region [2, 3]. However, using them for a specific irrigated agro-landscape should consider the spatial variability of natural conditions, primarily soil, geomorphological, hydrogeological, which is quite large in our natural and climatic zone (Figure 1).

Improving the efficiency of irrigated land is achieved by introducing modern technical solutions, namely automated control systems for sprinklers that help change irrigation rates in real time depending on the level of actual moisture reserves of the field and develop digital technology for determining the optimal parameters of soil moisture, differentiated by phases of growth and development of cultivated crops and taking into account the water-physical features of soil horizons of irrigated fields.

One of the most effective methods of reducing water supply and at the same time saving irrigation water, the most important natural resource, is the use of irrigation regimes, differentiated by the pre-irrigation moisture threshold and the calculated depth of the moistened soil throughout the growing season. It is known that the use of differentiated irrigation regimes according to the pre-irrigation humidity threshold saves irrigation water $450-650 \mathrm{~m}^{3} / \mathrm{ha}$, and for some crops, for example, soybeans - up to $1080 \mathrm{~m}^{3} / \mathrm{ha}$.

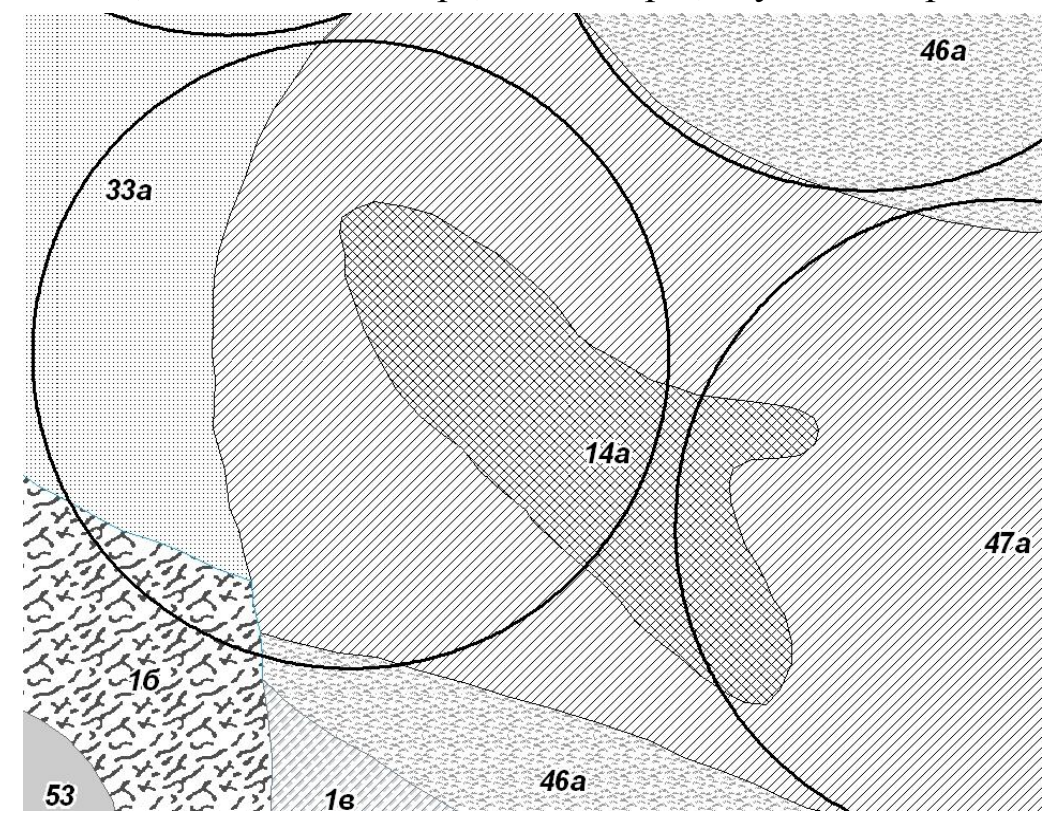

Fig. 1. Spatial variability of irrigated soils

1b, c - Dark brown medium heavy- and medium-loamy soils

14a - Solodic clay alkali soils

33 a - Dark brown alkalinized in complex with alkali soils, $25-50 \%$ clay

46a - Meadow brown alkalinized in complex with alkali soils, $10-25 \%$ clay

$47 \mathrm{a}$ - Meadow brown alkalinized in complex with alkali soils, $30 \%$ clay

53 - Sands

Due to the fact that the main parameters that determine the value of the irrigation rate are the power of the moistened soil layer and its pre-irrigation humidity, arising from 
the biological characteristics of cultivated crops in different phases of growth and development, as well as density and humidity at the lowest moisture content differing for different soil differences and particle size distribution, their incorrect choice can lead to drying of the root zone, respectively, the loss of productivity of irrigated crops, and excessive water supply, and finally to unproductive losses of irrigation water, its filtration into groundwater systems of the Saratov region $15-20 \%$ of the total water supply for irrigation. Technological advances that contribute to the growth of data collection and analysis capabilities, along with the improvement of methods of their analysis, can significantly increase the efficiency of irrigation. Artificial intelligence techniques (artificial neural networks, fuzzy logic, machine learning algorithms, etc.) are increasingly used for the management of agro-bio-systems in general and irrigation in particular. An overview of such methods is presented in paper [4]. Papers [5] and [6] deal with the applications of the above-mentioned methods for increasing the efficiency of irrigation. New tools for data mining, in-depth learning, etc. provide improved accuracy by resolving complex relationships in large amounts of parameters and have great potential. Thus, on their basis, decision support systems in irrigated agriculture are developed [7]. In this regard, it is advisable to conduct theoretical and experimental studies in the direction of modeling the optimization of water use in the irrigation area using the methods of neural network data analysis based on Kohonen neural networks and GIS technologies.

\section{MATERIALS AND METHODS}

One of the key issues of forecasting water use in the irrigation area is the amount of data, their incompleteness and uncertainty, as well as the large dimensionality of the factors influencing the amount of water supply. The solution to these problems is the use of data mining technologies. Namely, the use of neural networks in conjunction with methods and models of statistical modeling and forecasting. The scientific novelty of the study is the development of a methodology for predicting water use in the irrigation area based on Kohonen neural networks.

An irrigation system with wide-span circular sprinklers can be schematically represented as follows (Figure 2):

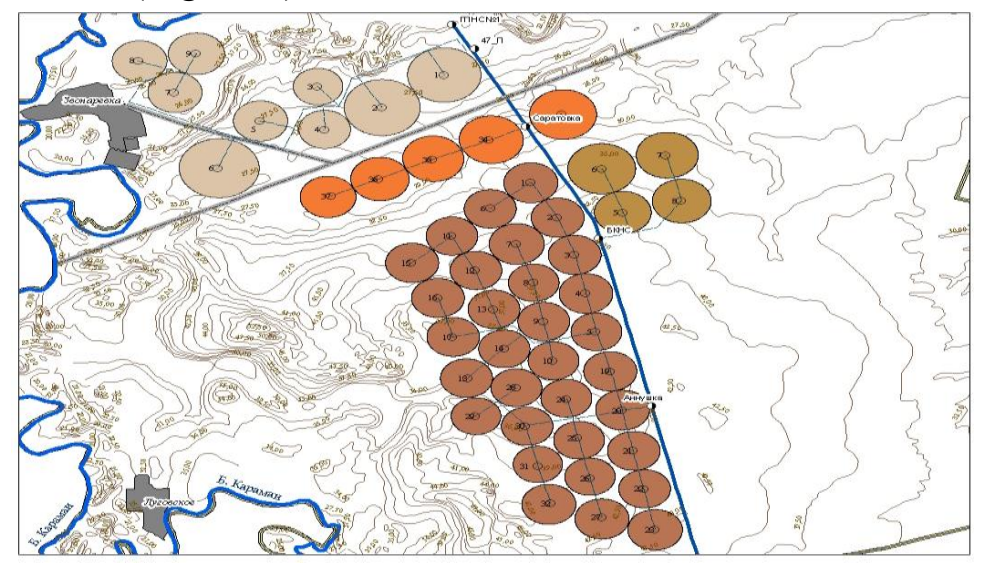

Fig. 2. An irrigation system with wide-span circular sprinklers 
As the figure shows, each sprinkler is located on a specific site with certain geocoordinates, which determines the presence of spatial variability of soil, geomorphological, hydrogeological conditions. The amount of water supply $(M)$ at the irrigation site depends on the value of irrigation rate $(m)$, determined by the biological characteristics of the cultivated crop $\left(X_{1}\right)$ in different phases of growth and development, as well as the power of the moistened soil layer $\left(X_{2}\right)$ and its pre-irrigation humidity $\left(X_{3}\right)$ addition $\left(X_{4}\right)$ and moisture $\left(X_{5}\right)$ at the lowest moisture content of moistened soil horizons. This value is the sum of the water consumption values of each sprinkler.

$$
M=M_{1}+M_{2}+\ldots+M_{\mathrm{n}} \text { or } M=n M_{\mathrm{i}},
$$

as site irrigation planning neglects, as a rule, geospatial variability of conditions. The application of classical forecasting methods in this case consists in constructing, for example, a multiple linear regression equation describing the relationship of the values of each sprinkler irrigation rate to factors $\left(X_{1}-X_{5}\right)$.

$$
m_{\mathrm{i}}=a_{1} X_{1}+a_{2} X_{2}+\ldots+a_{5} X_{5}
$$

However, this approach gives rise to at least two significant problems: the first - each of these factors depends on the totality of the remaining. For example, the pre-irrigation humidity $\left(X_{2}\right)$ depends on the characteristics of the cultivated crop in different phases of growth and development and therefore the factor $X_{2}$ is the vector $X_{2}=\left(X_{21}, X_{22}, \ldots\right.$, $X_{2 \mathrm{j}}$ ) for each crop, i.e. the dimension of equation (2) increases significantly. The second problem is the actual ignoring of the spatial variability of soil, geomorphological, hydrogeological conditions. Thus, the solution to these problems requires the development of a more accurate methodology for forecasting water use in the irrigation area using the latest developments in the field of artificial intelligence, namely the use of neural networks and self-organizing Kohonen maps. Assuming, without loss of generality, that the cultivated crop and its growth phase are fixed at the time of construction of the model, we will focus the study on the variation of geospatial variability of conditions in the irrigation area.

The idea of a Kohonen self-organizing map belongs to the Finnish scientist Toivo Kohonen [8]. Kohonen self-organizing maps are a type of artificial neural network that allows nonlinear regression and displays multidimensional data on a two-dimensional plane while preserving distances in the original data space [8]. This is one of the types of neural networks that use uncontrolled learning. In such learning, the learning set consists only of the values of the input variables, in the learning process there is no comparison of the outputs of neurons with the reference values. We can say that such a network learns to understand the structure of data.

Key applications of Kohonen maps include intelligence analysis and detection of new phenomena. In the case of exploratory data analysis, the Kohonen network can recognize clusters in the data, as well as establish the proximity of classes, which improves the understanding of the data structure to refine the neural network model ([9]). The Kohonen network recognizes clusters in learning data and assigns all data to 
one or another cluster. If the network then detects data sets that do not resemble any of the known samples, it reveals its novelty. The use of self-organizing maps allows us to analyze the features of the cluster structure of multidimensional sampling, which is important for qualitative analysis of information in our case. A schematic representation of the structure of the Kohonen network is presented in Figure 3.

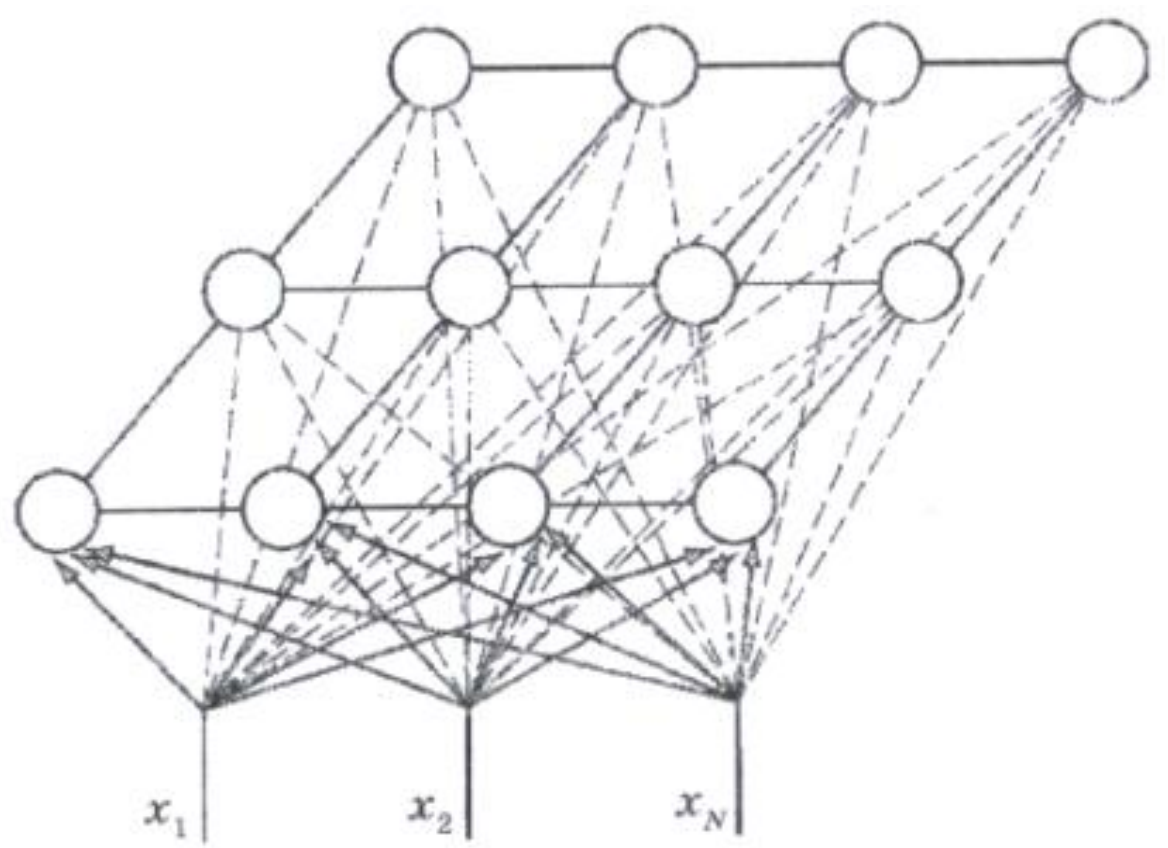

Fig. 3. Schematic representation of the structure of the Kohonen network

As the figure shows, the Kohonen network is quite simple and consists of two layers: input and output. Map elements are located in some space, usually two-dimensional. As the Kohonen network belongs to self-organizing networks, when it receives input signals, it does not receive information about the desired output signal. This leads to the impossibility of forming a tuning criterion based on the mismatch of the actual and required output signals of the neural network, so the weight parameters of the network are adjusted based on other algorithms. All the presented input signals from a given learning set, the self-organizing network in the learning process divides into classes, building so-called topological maps. Kohonen network is trained by the method of successive approximations. In the process of learning such networks, data is fed to the inputs, but the network is not adjusted to the reference value of the output but to the patterns in the input data. Training begins with a randomly selected output location of the centers. In the process of sequential feeding of a network of learning examples to the input, the most similar neuron is determined (the one that has a scalar product of the scales and the vector fed to the input is minimal). This neuron is declared the winner and is the center for adjusting the scales of neighboring neurons. Such a learning rule assumes "competitive" learning, considering the distance of neurons from the "winning 
neuron". The learning is not to minimize the error, but to adjust the scales (internal parameters of the neural network) for the greatest coincidence with the input data. The scheme and mathematical implementation of the Kohonen network learning algorithm are well developed [], [] and can be briefly presented in the following form (Figure 4).

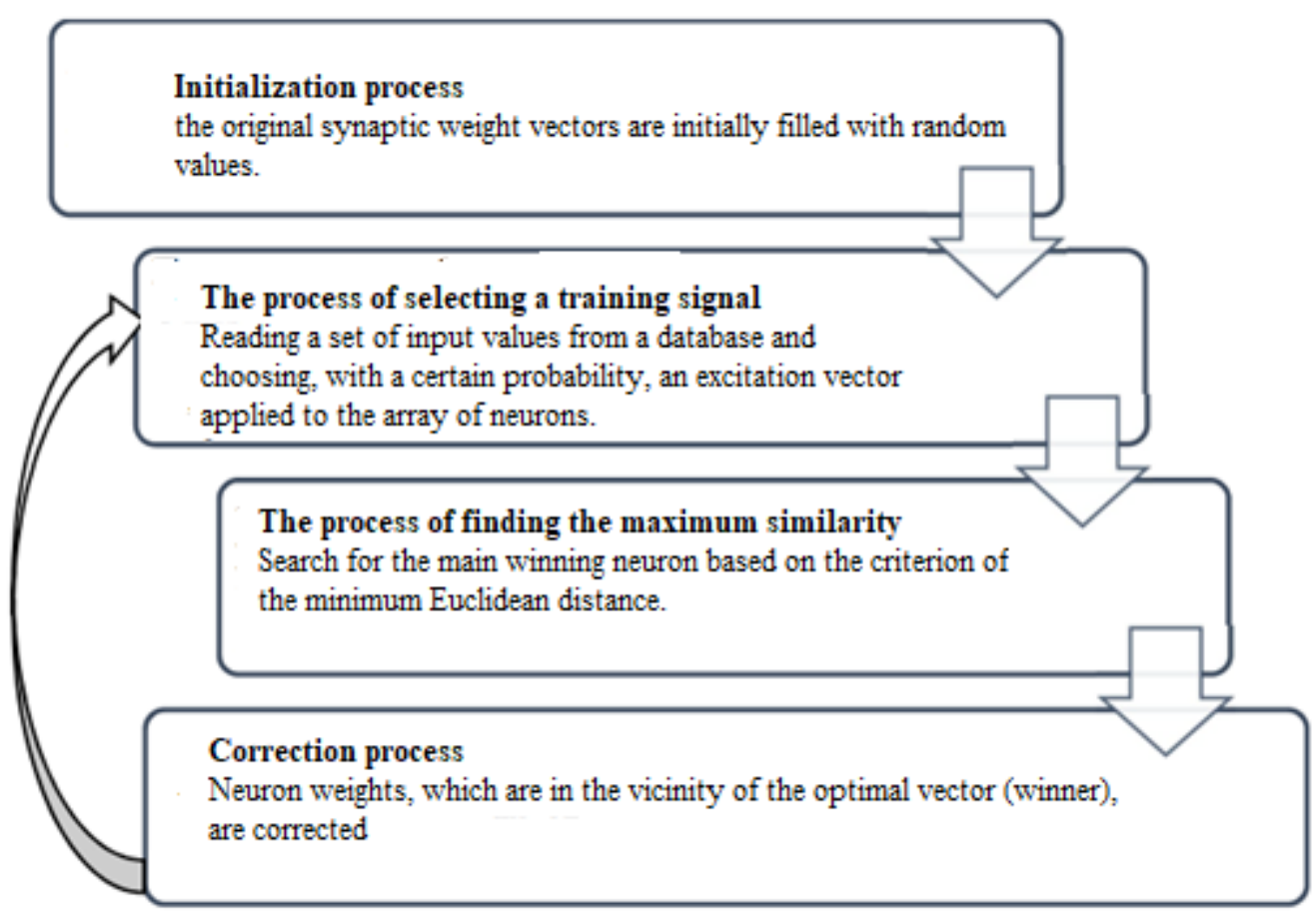

Fig. 4. Kohonen network learning algorithm

The steps of the algorithm are repeated until the output values of the network are stabilized with the specified accuracy. An important point in the projection of multidimensional data on the plane is its quality achieved in the Kohonen network at several levels: preservation of the topology (i.e., on many sets of source data and neurons of the trained network, the neighborhood structure is the same), preservation of order between equivalent pairs of points are proportional) and the preservation of metric properties when compressing space [8]. The "projection screen" as a result of training acquires the properties of an ordered structure in which the values of the synapses of neurons change smoothly along two dimensions. The color and location of the two-dimensional lattice fragments is used to analyze the patterns associated with the components of the data set. Each feature generates its own coloring of the cells of the map by the value of the average value of this feature in the data included in this cell. Combining maps of all the features of interest to us, we obtain a topographic atlas that gives an integrated idea of the structure of multidimensional data. 


\section{RESULTS AND DISCUSSION}

As data for modeling, the data of the geoinformation system of monitoring of the irrigated lands of CJSC Agrofirma Volga, Saratov region, Russia are considered. According to agro-climatic conditions, the monitoring area is the zone of insufficient and unstable humidity, the climate is moderately continental with a predominant number of clear and cloudy days a year, hot and dry summers, cold and snowless winters, short springs and short autumns. High values of temperature occur in June, July and August, the average maximum in July $+30^{\circ} \mathrm{C}$, low in January and February, the average minimum in February $-23^{\circ} \mathrm{C}$. Spring is unstable, the cold lasts until April, the snow cover reaches $30-40 \mathrm{~cm}$. Summer is hot, droughts are possible, as well as a sharp cooling at the beginning and end. The maximum amount of precipitation is 49 $\mathrm{mm}$ in November, the minimum is $25 \mathrm{~mm}$ in April, the precipitation is unevenly distributed. The relative humidity is maximum in winter and reaches $85 \%$, the minimum value - in summer $55 \%$.

Analysis of the geoinformation data on monitoring of irrigated lands of CJSC Agrofirma Volga [62] showed that they are located on 131 contours of 18 soil differences. Moreover, individual irrigated areas are located on several soil differences - from 2 to 5 (Figure 5).

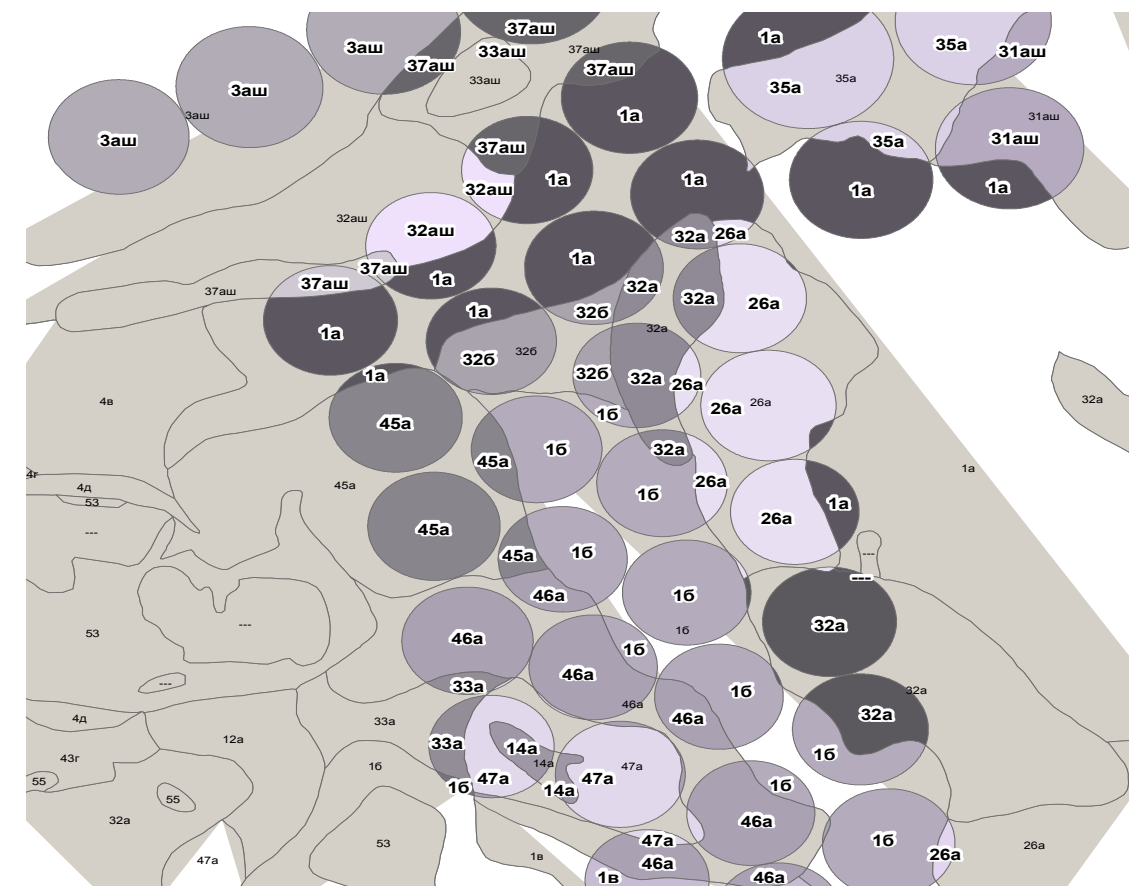

Fig. 5. A fragment of the layer of the "Irrigated soils" digital map, combined with the layer of soil differences of the reclamation complex of CJSC Agrofirma Volga of the Marx district of the Saratov region

1a, b, c - Dark brown medium heavy- and medium-loamy clay soils

Заш - Dark brown alkalinized clay soils, chocolate clay-based

14a - Solodic clay alkali soils 
26a - Dark brown medium clay, in a complex with alkali soils 5-10\%

31 aw - Dark brown alkalinized clay soils, chocolate clay-based, in a complex with deep alkali soils $25-50 \%$

32a - Dark brown alkalinized clay soils in complex with alkali soils, 10-25\%

33 a - Dark brown alkalinized clay soils in complex with alkali soils, $25-50 \%$

$35 \mathrm{a}$ - Dark brown thin clay soils in complex with deep alkali soils 5-10\%

37 aw - Dark brown alkalinized clay soils, chocolate clay-based, in a complex with alkali soils $10-25 \%$

45a - Meadow brown alkalinized clay soils in complex with alkali soils, 5-10\%

46a - Meadow brown alkalinized clay soils in complex with alkali soils, 10-25\%

$47 \mathrm{a}$ - Meadow brown alkalinized clay in complex with alkali soils, 30\%

53 - Sands

The irrigated territory of CJSC Agrofirma Volga is represented by eighteen main soil differences from sands to clay alkali soils, with the main share of dark chestnut and meadow-chestnut of various alkali degree and in a complex with alkali soils. The diagram of soil differences in the section of the total occupied area is presented in Figure 6.

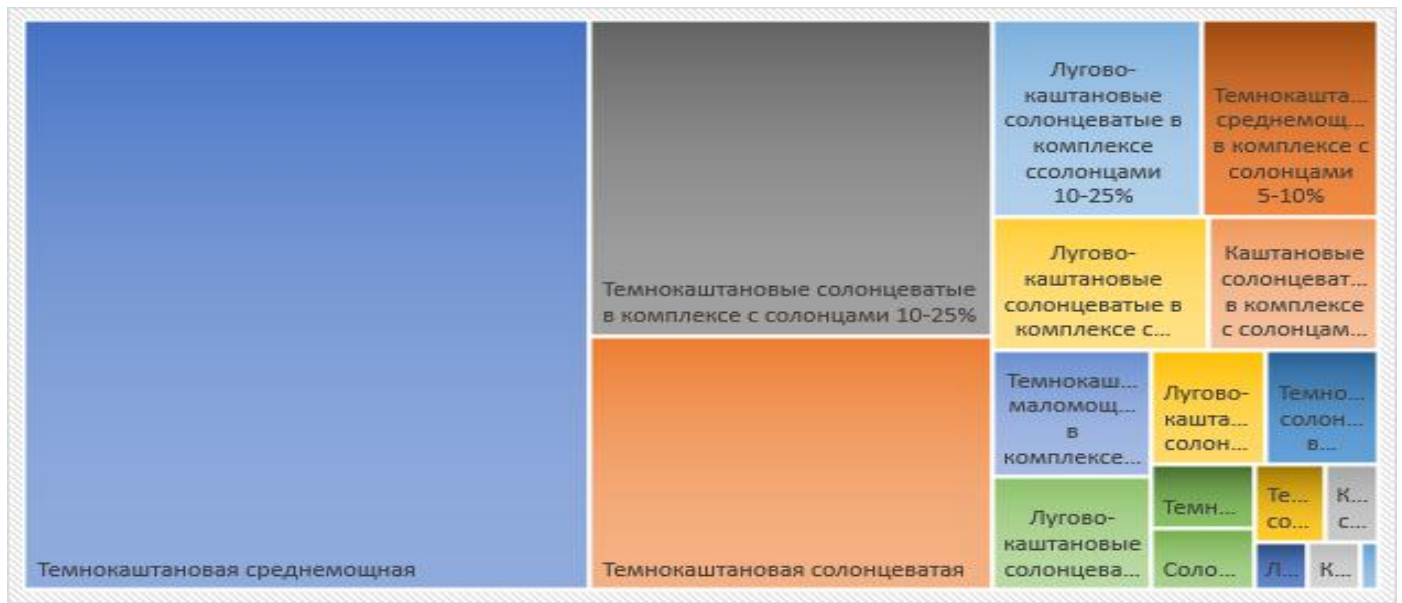

Fig. 6. Soil differences of the irrigated territory of CJSC Agrofirma Volga

The presence of such a large number of soil differences in terms of irrigation practice does not allow for accurate differentiation of irrigation rates in the irrigation area. We apply the neural network modeling algorithm in the form of a Kohonen self-organizing map to display multidimensional data of soil differences on a two-dimensional plane while preserving distances in the original data space. Let us consider the model 
precisely in terms of geospatial variability of soil conditions.

We will feed data to the network input for each landfill:

Table 2. Data structure

\begin{tabular}{|c|c|c|c|}
\hline $\begin{array}{c}\text { Landfill } \\
\text { No. }\end{array}$ & $\begin{array}{c}\text { Dark } \\
\text { brown } \\
\text { medium } \\
X_{1}\end{array}$ & $\begin{array}{l}\text { Dark brown alkalinized } \\
\text { soils in complex with } \\
\text { alkali soils, } 10-25 \% \\
\qquad X_{2}\end{array}$ & $\begin{array}{c}\text { Meadow brown } \\
\text { alkalinized in complex } \\
\text { with alkali soils, } 30 \% \\
X_{18}\end{array}$ \\
\hline $\mathrm{i}$ & $a_{\mathrm{i} 1}$ & $a_{\mathrm{i} 2}$ & $a_{\mathrm{i} 18}$ \\
\hline
\end{tabular}

Where aij is a sign of the $\mathrm{j}$-th soil difference at the $\mathrm{i}$-th polygon. Our task will be to cluster these soil differences to an acceptable dimension (1-3), which allows, on the one hand, to consider the characteristic properties in terms of optimizing irrigation rates, and on the other hand, to make the algorithm acceptable for practical use. Let us consider the most represented soil varieties in this area - Dark brown medium (Input 1), Dark brown alkalized in a complex with alkali soils 10-25\% (Input 2), Dark brown alkalinized (Input 3) and Meadow brown alkalinized in a complex with alkali soils 5$10 \%$ (Input 4 ) in terms of the occupied area.

We will simulate Kohonen self-organizing maps in Matlab [10]. The network architecture and learning process are shown in Figure 7.

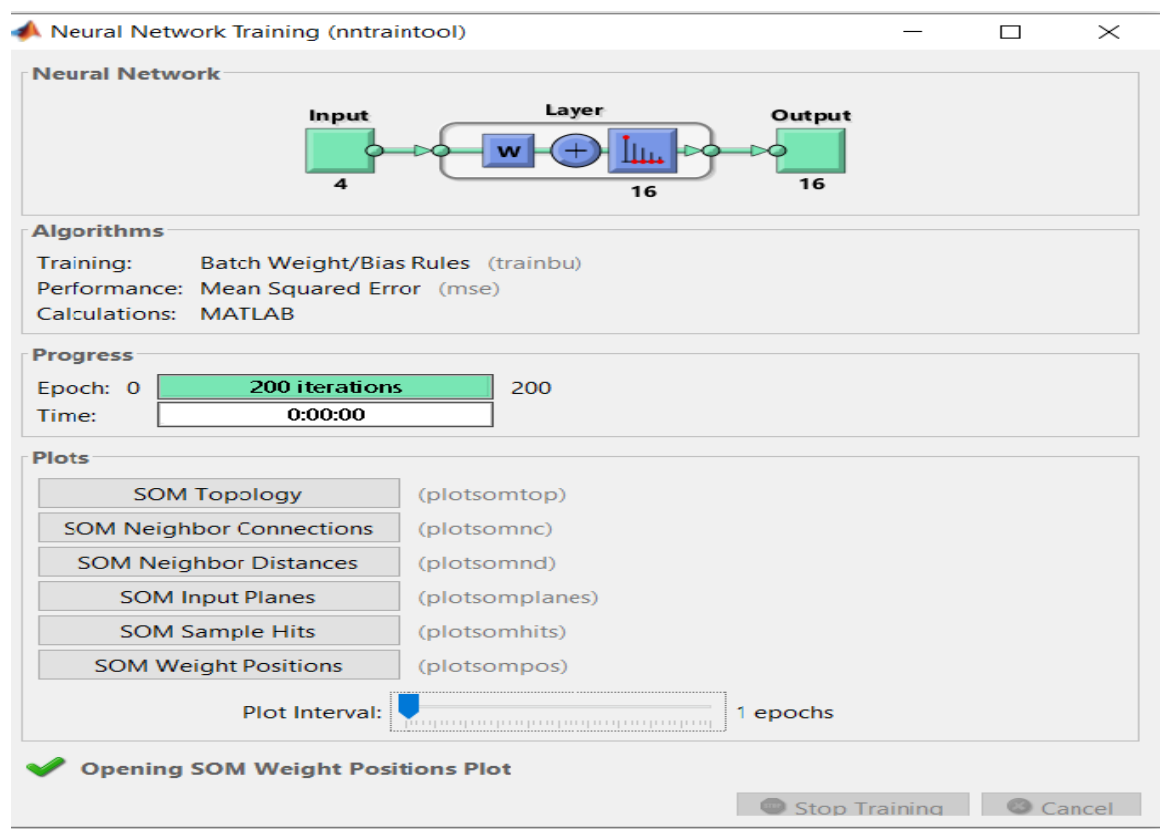

Fig. 7. Network architecture and learning process in Matlab

We obtain the following characteristics of the object of study: the location of neurons in the topology and the amount of training data associated with each of the neurons the centers of the clusters (Hits), the distances between neighboring neurons (SOM 
Neighbor Weight Distances) (Figure 8) and visualization of the weights of each $-\mathrm{i}-\mathrm{th}$ feature (Weights from Input i), which connect each input to each of the neurons (Figure 9).
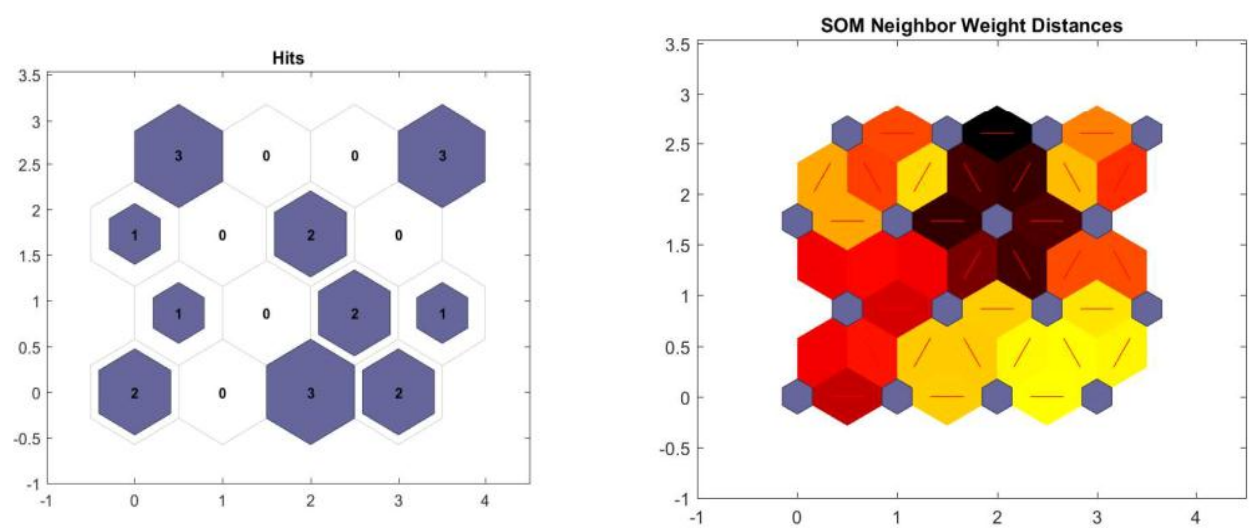

Fig. 8. Diagrams of location (left) and distance (right) between neurons
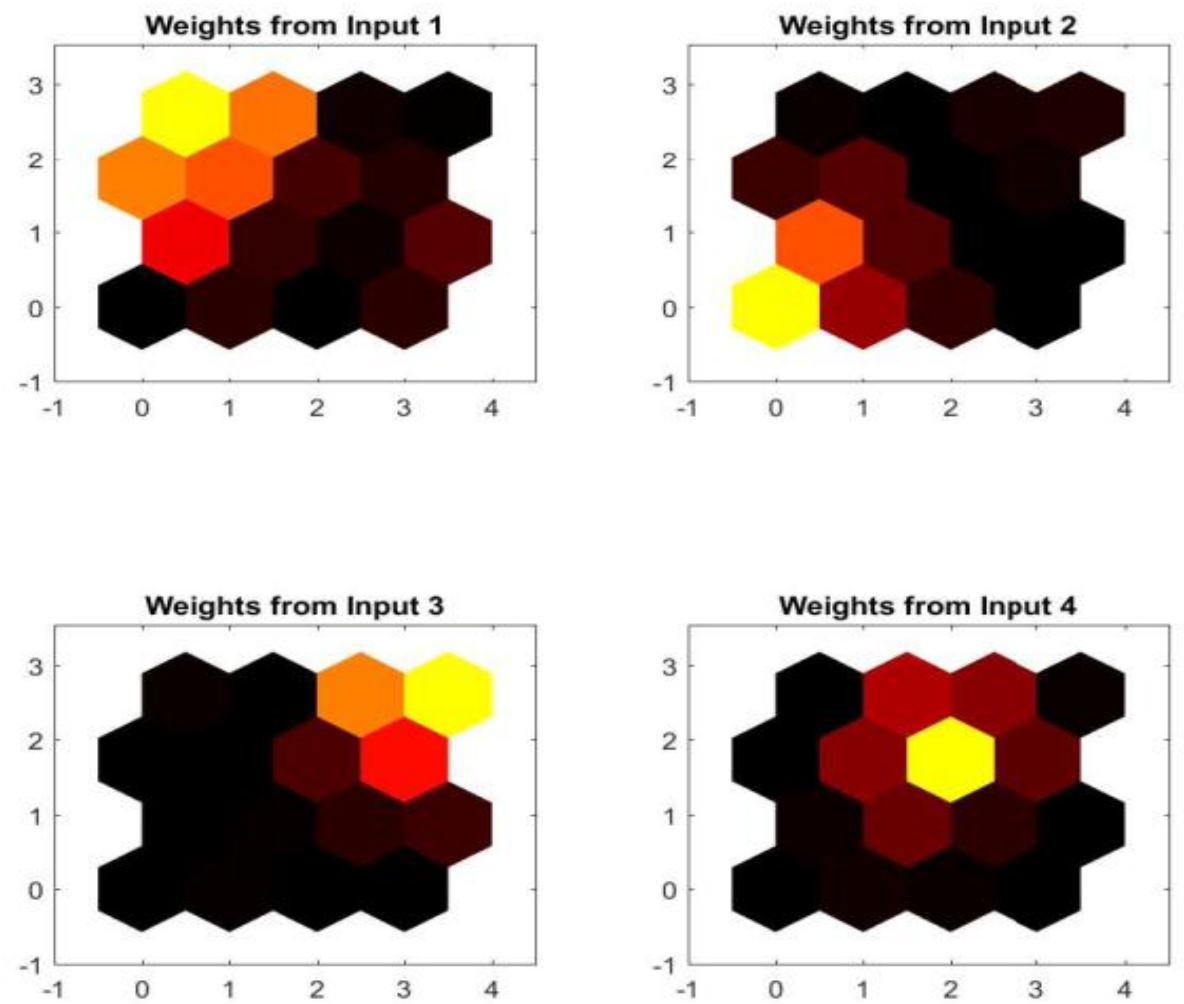

Fig. 9. Visualization of weights of four types of soil differences

In this example, the data is slightly more concentrated in the lower right neurons but 
the distribution is fairly even in general. The figure of SOM Neighbor Weight Distances shows the distances between adjacent neurons. Blue hexagons represent neurons. Red lines connect adjacent neurons. The colors in the areas containing the red lines indicate the distances between neurons. Darker colors represent longer distances. Lighter colors represent shorter distances. As can be seen from the presentation, the SOM network appears to have grouped soil differences into two distinct groups. Further analysis of the weights (Figure 2) shows that each element of the input vector (in this case, four) there is a plane of weights. They are visualizations of the weights that connect each input to each of the neurons (lighter and darker colors represent more and less weights, respectively). The performed neural filtering makes it possible to reveal deeper connections between the studied parameters. The most topologically connected in this case will be the first and second features, while the third and fourth are weakly correlated with them. That is, when differentiating irrigation rates in the irrigation area, it is enough to consider two soil varieties, namely Dark brown medium and Dark brown alkalinized in combination with $10-25 \%$ alkali soils. This is one of the aspects of using Kohonen self-organizing maps. It is possible to carry out both a deeper cluster analysis of the irrigated area and the application of the results obtained at the stage of planning the landfills of the irrigated land, namely the selection of landfills with the most topologically similar features.

\section{CONCLUSION}

The presence of a large number of soil differences does not allow for accurate differentiation of irrigation rates in the irrigation area. A model of geospatial variability of soil conditions is considered. We apply the neural network modeling algorithm in the form of a Kohonen self-organizing map to display multidimensional data of soil differences on a two-dimensional plane while preserving distances in the original data space. Let us consider the model precisely in terms of geospatial variability of soil conditions.

The neural network model makes it possible to reveal deeper connections between the studied parameters. To determine the parameters of differentiation of irrigation rates at the new irrigation area, a vector with the coordinates of the features of this area should be fed to the input of the trained network. The network will determine the cluster to which this vector can be attributed, which will allow more accurate differentiation of irrigation rates, thereby optimizing the value of the irrigation rate issued by each sprinkler $m_{\mathrm{i}}$ and, accordingly, the amount of water consumption by each sprinkler Mi. The neural network model is quite simple to implement, compared to classical regression methods, and allows you to take into account a large number of factors in addition to soil conditions.

For medium-term forecasts, it is proposed to use the Kohonen map, which, once superimposed on a geoinformation map, allows building not only a theoretical forecast, but also a visual representation of this forecast. The network learns in the period of time T. Then, in the period of time $(T+1)$, polygons with the values of indicators in the period $(T+1)$ are fed to the same network (without training) and their position on the 
map is determined, as a result, you can answer the question, to which cell on the map a certain polygon will belong now. This fact can serve as a basis for making management decisions. In addition, by modeling various trends in water use (economic, environmental, etc.), it is possible to change external factors, changing the level and directions of optimization of water use.

\section{REFERENCES}

1. O. Gagatova, Report on the state and use of agricultural land in the Russian Federation in 2018 (M.: FSBSI "Rosinformagrotech", 2020)

2. N.A. Pronko, V.V. Korsak, A.S. Falkovich, Melioration and water management, 4 (2014)

3. V.A. Shadskikh, L.G. Romanova, V.E. Kizhaeva, Melioration and water management, 6 (2017)

4. A. Kamilaris, A. Kartakoullis, FX. Prenafeta-Boldú, Computers and Electronics in Agriculture, 143 (2017)

5. B.A. King, K.C. Shellie, Agricultural Water Management, 167 (2016)

6. H. Navarro-Hellin, J. Martinez-del-Ricon, R. Domingo-Miguel, F.Soto-Valles, R. Torres-Sances, Computers and Electronics in Agriculture, 124 (2016)

7. X. Song, G. Zhang, F. Liu, D. Li, Y. Zhao, J. Yang, Journal of Arid Land, 8 (2016)

8. T. Kohonen, Self-Organizing Maps (BINOM Publishing house. Laboratory of Knowledge, 2014)

9. Cheng-Jian Lin, Chun-Cheng Peng, Cheng-Hung Chen, and Hsueh Yi Lin, Applied Mathematics \& Information Sciences, 9 (2015)

10. M. Beale, M. Hagan, H. Demuth, Neural Network ToolboxTM User's Guide (The Math Works, Inc, 2015) 